\title{
ORIGINAL
}

\section{ANÁLISIS COSTE-EFECTIVIDAD DEL TRATAMIENTO DE MANTENIMIENTO CON RITUXIMAB EN PACIENTES CON LINFOMA FOLICULAR QUE RESPONDEN A LA TERAPIA DE INDUCCIÓN EN PRIMERA LÍNEA (*)}

\author{
Antonio J Castro Gómez (1), Armando López-Guillermo (2), Antonio Rueda Domínguez (3), Antonio \\ Salar (4), Cristina Varela Moreno (1) y Carlos Rubio-Terrés (5).
}

(1) Roche Farma. Madrid.

(2) Hospital Clínico. Barcelona.

(3) Hospital Costa del Sol. Marbella. Málaga.

(4) Hospital del Mar. Barcelona.

(5) Health Value. Madrid.

(*) Estudio realizado con una ayuda a la investigación, sin restricciones, de Roche Farma, SA.

\begin{abstract}
RESUMEN
Fundamentos: El tratamiento de mantenimiento con rituximab de linfoma folicular (LF) que responde a la inducción en primera línea, aumenta de manera significativa la supervivencia libre de progresión en comparación con la observación. Para evaluar económicamente esta opción terapéutica, se realizó un análisis de coste-efectividad del tratamiento de mantenimiento del LF tras la inducción en primera línea con rituximab frente a la observación de la enfermedad.
\end{abstract}

Métodos: Se hizo un modelo de Markov del LF, con cuatro estados de salud (supervivencia libre de progresión en primera o segunda línea, progresión y muerte). Las probabilidades de transición entre los estados se obtuvieron de los ensayos clínicos PRIMA y EORTC 20981. Las utilidades de los estados se obtuvieron de la bibliografia. El uso de recursos sanitarios, desde la perspectiva del Sistema Nacional de Salud, fue estimado por un panel de expertos clínicos españoles. Los costes unitarios ( $€$ del año 2011) se obtuvieron de fuentes españolas. Se efectuaron análisis determinísticos y probabilísticos.

Resultados: En el caso base del análisis determinístico, para un horizonte temporal de 30 años, el coste por año de vida ganado (AVG) y por año de vida ajustado por calidad (AVAC) ganado, fue de $5.663 €$ y $6.253 €$, respectivamente. Los análisis de sensibilidad confirmaron la estabilidad del caso base para horizontes temporales de 10 y 20 años y diversas distribuciones estadísticas (Weibull, exponencial, log-logística, log-normal, Gompertz y gamma) oscilando entre 4.222 y $8.766 €$. El mantenimiento con rituximab es coste-efectivo a partir de un horizonte temporal de 5,7 años (coste por AVAC ganado de 29.998 €).

Conclusión: En comparación con la observación, el tratamiento de mantenimiento con rituximab del LF que responde a la inducción en primera línea, es coste-efectivo de acuerdo con el presente modelo.

Palabras clave: Análisis coste-beneficio. Rituximab. Observación. Linfoma folicular.

Correspondencia

Carlos Rubio Terrés

Health Value

Health Economics and Research of Outcomes Consulting.

C/ Virgen de Aránzazu, 21. 28034 - Madrid.

Correo electrónico: crubioterres@healthvalue.org

\section{ABSTRACT}

\section{Cost-Effectiveness Analysis of Maintenan- ce Therapy with Rituximab in Patients with Follicular Lymphoma Responding to Induction Therapy at the First Line}

Background: Maintenance therapy with rituximab for follicular lymphoma (FL) responding to induction at the first-line, significantly increases progression-free survival compared with observation. To estimate the efficiency of this therapeutic option, we performed a cost-effectiveness analysis of maintenance therapy of the follicular lymphoma (FL) that responds to induction in first line, with rituximab, compared with the option of "watch and wait" strategy.

Methods: We did a Markov model of the FL, with four health states (progression free survival in first or second line, progression and death). The transition probabilities between states were obtained from clinical trials PRIMA and EORTC 20981. Health state utilities were obtained from literature. The use of health resources, from the perspective of the National Health System was estimated by a panel of Spanish clinical experts. Unit costs ( $€$ in 2011) were obtained from Spanish sources. Deterministic and probabilistic analyses were made.

Results: In the deterministic base case analysis, for a time horizon of 30 years, the cost per life year gained (LYG) and quality-adjusted lifeyears (QALYs) gained, was $€ 5,663$ and $€ 6,253$ respectively. The sensitivity analyses confirmed the stability of the base case for time horizons of 10 and 20 years and various statistical distributions (Weibull, exponential, log-logistic, log-normal, Gompertz, and gamma) ranging between $€ 4,222$ and $€ 8,766$. Rituximab maintenance is cost-effective from a time horizon of 5.7 years (cost per QALY gained of $€ 29,998$ )

Conclusion: Compared with observation, rituximab maintenance treatment of the FL that responds to induction therapy in first line, is cost-effective according to the present model.

Key words: Cost-benefit analysis. Rituximab. Observation. Lymphoma Follicular. 


\section{INTRODUCCIÓN}

El linfoma no Hodgkiniano folicular (LF) es una neoplasia asociada con las células B del centro germinal del ganglio linfático. Es el segundo subtipo de linfoma más frecuente, con una tasa de incidencia de 5-7 casos por 100.000 personas. Los LF se caracterizan por su curso indolente, con medianas de supervivencia prolongadas (8-10 años $)^{1}$. La mediana de supervivencia con las terapias actuales es de 14 años ${ }^{2}$.

A pesar de la elevada tasa de respuesta al tratamiento inicial, la mayoría de los pacientes recaen después del mismo. Aproximadamente el $80 \%$ son diagnosticados con enfermedad avanzada (estadios III o IV $)^{3}$ considerándose que son incurables con los tratamientos convencionales, por lo que el objetivo de éstos es conseguir la "paliación óptima" cuyo fin es prolongar la supervivencia y conseguir períodos de tiempo prolongados en los que el paciente se encuentre libre de síntomas relacionados con la enfermedad o el tratamiento ${ }^{4}$.

Teniendo en cuenta que la edad media de los pacientes con LF está entre los 60 y los 65 años y el curso natural de la enfermedad, con remisiones espontáneas entre el 15-20\% de los casos, en algunos pacientes asintomáticos se toma una actitud conservadora de observación (o "espera vigilante”) ${ }^{1}$.

En un intento de variar el curso clínico de la enfermedad, una estrategia terapéutica diferente de los linfomas es la administración de un tratamiento de mantenimiento después de haber conseguido una respuesta adecuada. Este tratamiento de mantenimiento debe entenderse como parte de una estrategia integral en la terapéutica de estos pacientes.

Durante los últimos años, la introducción de una nueva opción terapéutica, la de la inmunoterapia con anticuerpos monoclonales, ha permitido aumentar la especificidad del tratamiento, reduciendo la toxicidad y presentando además sinergismo con la quimioterapia convencional debido a su distinto mecanismo de acción ${ }^{5}$. Así, hoy en día se ha demostrado que la mejor terapia para pacientes con LF con criterios de tratamiento es la combinación de Rituximab, primer anticuerpo monoclonal quimérico murino/humano frente al antígeno CD20 para el linfoma no hodgkiniano, con poliquimioterapia ${ }^{6}$. Más recientemente, los resultados del ensayo clínico PRIMA (Primary RItuximab and MAintenance) indican que el tratamiento de mantenimiento con rituximab del LF que responde a la inducción en primera línea (esquemas R-CHOP, R-CVP o $\mathrm{R}-\mathrm{FCM}$ ) aumenta la supervivencia libre de progresión, con un hazard ratio de 0,55 (IC95\% 0,44; 0,68; $\mathrm{p}<0,0001$ ) en comparación con la observación ${ }^{7}$. De este modo, el mantenimiento con rituximab se ha convertido en una indicación terapéutica formal. Sin embargo, son todavía escasos los estudios fármacoeconómicos que valoren exactamente el impacto económico y el beneficio clínico real de esta nueva aproximación terapéutica.

En este contexto, el objetivo de este estudio es realizar un análisis coste-efectividad del tratamiento de mantenimiento en primera línea del LF con rituximab frente a la opción de observación de la enfermedad.

\section{MATERIAL Y MÉTODOS}

Modelo fármacoeconómico. El estudio consistió en un modelo fármacoeconómico que permite hacer simulaciones de procesos sanitarios complejos relacionados con medicamentos y que, siguiendo un protocolo previamente establecido, es elaborado mediante estimaciones obtenidas a partir de los datos disponibles de eficacia, toxicidad y costes de las alternativas comparadas $^{8}$. Se hizo la adaptación al sistema sanitario español de un modelo 
internacional de Markov ${ }^{9,}{ }^{10}$, con una estructura que se representa en la figura 1 y que se describe en detalle más adelante.

Población diana. Representa el conjunto hipotético de pacientes en los que se realiza el análisis teórico $\mathrm{y}$, por tanto, la población a la que pueden aplicarse los resultados del estudio. La población diana fueron pacientes de ambos sexos, con diagnóstico de LF (de grados 1, 2 ó 3a) que respondieron a la inducción en primera línea con los esquemas R-CHOP, R-CVP o $\mathrm{R}-\mathrm{FCM}$, conforme a las características de los pacientes del ensayo clínico PRIMA, cuyos resultados se utilizaron en el modelo fármacoeconómico (tabla 1$)^{7}$.

Estados de salud. De acuerdo con la historia natural del LF, en el modelo se consideraron los siguientes estados de salud (figura 1): tres estados "transitorios" (supervivencia libre de progresión en primera línea de tratamiento [SLP-1L] o en segunda línea [SLP-2L] y progresión de la enfermedad [Progresión]) en los que los pacientes podrían permanecer varios ciclos de 1 mes de duración, y el denominado estado "absorbente" (la muerte de los pacientes) (figura 1). Todos los pacientes de la cohorte estarían inicialmente libres de progresión en primera línea. A lo largo de los ciclos mensuales, los pacientes pueden seguir en ese estado (SLP-1L) o bien transitar a los otros tres (SLP-2L, progresión y muerte). Una vez se produce la progresión, el paciente puede permanecer en ese estado o morir.

El objetivo del modelo fue estimar las diferencias entre las opciones terapéuticas (mantenimiento con rituximab u "observación") comparadas en los siguientes aspectos: (i) los años de vida ajustados por su calidad (AVAC); (ii) los años de vida sin ajustar por su calidad (AV); (iii) el tiempo en el que los pacientes sobreviven sin progresión de la enfermedad; así como (iv) los costes asociados a los estados SLP-1L,
SLP-2L y progresión. No se consideraron los costes debidos a la muerte (M).

Datos de eficacia y tipos de análisis. El tipo de análisis farmacoeconómico que debe realizarse depende de que existan o no diferencias demostradas de eficacia o de toxicidad entre los tratamientos. Como se indicó anteriormente, el estudio PRIMA, cuyas características se resumen en la tabla 1, concluyó que el tratamiento de mantenimiento con rituximab del LF que responde a la inducción en primera línea (con los esquemas R-CHOP, R-CVP o RFCM) aumenta la supervivencia libre de progresión en comparación con observación ${ }^{7}$ (tabla 1).

Debido a que se han demostrado diferencias clínicas entre las opciones terapéuticas comparadas, se hizo un análisis de coste-efectividad (coste por año de vida ganado, AVG). Por otra parte, debido a que la diferente tasa de progresión de la enfermedad constatada entre los pacientes en mantenimiento con rituximab y los sometidos a observación, podría repercutir en la calidad de vida y, por tanto, en las utilidades expresadas como años de vida ajustados por calidad (AVAC), el coste-efectividad también se valoró como coste por AVAC ganado.

La comparación de los resultados incrementales de coste-efectividad se efectuó aplicando la fórmula siguiente:

Costes por paciente en mantenimiento con rituximab-costes por paciente en observación / AV o AVAC por paciente en mantenimiento con rituximab-AV o AVAC por paciente en observación

Los resultados se presentan como costes incrementales, AVG, AVAC ganados, coste por AVG y como coste por AVAC ganado con el mantenimiento con rituximab, en comparación con la opción de la "espera vigilante" u observación. 
Tabla 1

Principales premisas y asunciones tomadas en el análisis farmacoeconómico realizado mediante un modelo de Markov

\begin{tabular}{|c|c|c|c|c|c|c|}
\hline \multicolumn{6}{|l|}{ Ítem } & Referencia \\
\hline \multicolumn{6}{|c|}{ 1.Estados de salud del modelo: SLP-1L, SLP-2L, Progresión, Muerte (figura 1) } & 9 \\
\hline \multicolumn{6}{|c|}{ 2. Pacientes con linfoma folicular que responde a la terapia de inducción en primera línea } & 7 \\
\hline \multicolumn{6}{|c|}{ 3. Edad $(60,0$ años), peso corporal $(64,3 \mathrm{~kg})$ y estatura $(161,50 \mathrm{~cm})$ medios de la cohorte } & 9,37 \\
\hline \multicolumn{6}{|c|}{ 4. Pauta de mantenimiento con rituximab: $375 \mathrm{mg} / \mathrm{m} 2$ cada 8 semanas ( 2 años frente a observación) } & 7 \\
\hline \multicolumn{6}{|c|}{ 5. Probabilidades de transición consideradas en el modelo: } & 7,15 \\
\hline Transición (figura 1) & desde & hasta & previo & Rituximab & Observación & \\
\hline A & SLP-1L & SLP-1L & - & \multicolumn{2}{|c|}{$\mathrm{HR}=0,55(\mathrm{IC} 95 \% 0,44 ; 0,68)$} & \\
\hline \multirow[t]{2}{*}{$\mathrm{B}$} & SLP-1L & SLP-2L & RIT & $26 \%$ & $74 \%$ & \\
\hline & & & OBS & $49 \%$ & $51 \%$ & \\
\hline $\mathrm{C}^{*}$ & SLP-1L & Muerte & - & 0,0003 & 0,0002 & \\
\hline $\mathrm{D}^{*}$ & SLP-2L & SLP-2L & - & \multicolumn{2}{|c|}{0,00118} & \\
\hline \multirow[t]{2}{*}{$\mathrm{E}^{*}$} & SLP-2L & progresión & RIT & 0,01927 & 0,01850 & \\
\hline & & & OBS & 0,01664 & 0,05509 & \\
\hline $\mathrm{F}^{*}$ & progresión & muerte & - & 0,02305 & 0,05016 & \\
\hline \multicolumn{6}{|c|}{ 6.Duración del ciclo de transición: mensual, con corrección de medio ciclo } & 9 \\
\hline \multicolumn{6}{|c|}{$\begin{array}{l}\text { 7.Horizonte temporal del análisis: hasta la muerte o } 30 \text { años (se estima que solo el } 5 \% \text { de la cohorte } \\
\text { sobreviviria a ese periodo) }[10-30 \text { años }]^{*}\end{array}$} & 13 \\
\hline \multicolumn{6}{|c|}{$\begin{array}{l}\text { 8. Modelo de Weibull para la extrapolación de la permanencia en SLP o la transición a progresión o muerte, } \\
\text { en el horizonte temporal (cálculo de las probabilidades de transición) [distribuciones gamma, exponencial, } \\
\text { log-logistica, log-normal y Gompertz]*. }\end{array}$} & 14 \\
\hline \multicolumn{6}{|c|}{ 9. Mortalidad por todas las causas en España. } & 17 \\
\hline \multicolumn{6}{|c|}{$\begin{array}{l}\text { 10.Modelo de minimos cuadrados ordinarios para estimar la mortalidad desde el estado de progresión en } \\
\text { segunda línea }\end{array}$} & 16 \\
\hline \multicolumn{6}{|c|}{ 11. Utilidades de los estados: SLP-1L: 0,880; SLP-2L: 0,790; Progresión: 0,620 } & 11,12 \\
\hline \multicolumn{6}{|c|}{ 12. Perspectiva del análisis: Sistema Nacional de Salud } & 9 \\
\hline \multicolumn{6}{|c|}{$\begin{array}{l}\text { 13.PVL de rituximab (con descuento: } 100 \mathrm{mg}=229,90 € ; 500 \mathrm{mg}=1.149,28 € \text { ) } \\
\text { [sin descuento: } 100 \mathrm{mg}=250,81 € ; 500 \mathrm{mg}=1.247,00 €)]^{*}\end{array}$} & 36 \\
\hline \multicolumn{6}{|c|}{$\begin{array}{l}\text { 14. Coste por paciente de la inducción con los esquemas de quimioterapia (excluido rituximab): } \\
\mathrm{CHOP}=27,43 € \quad \mathrm{CVP}=10,44 € \quad \mathrm{FCM}=238,70 €\end{array}$} & $31-36$ \\
\hline \multicolumn{6}{|c|}{$\begin{array}{l}\text { 15. Coste de la administración de rituximab: proporcional a la duración de la infusión IV en el hospital de dia: } \\
273,27 €[ \pm 20 \%]^{*}\end{array}$} & $22-30$ \\
\hline
\end{tabular}




\section{Tabla 1 (continuación)}

\begin{tabular}{|c|c|}
\hline $\begin{array}{l}\text { 16. Coste de los efectos adversos de los tratamientos: según la frecuencia observada en el ensayo clínico y } \\
\text { los costes unitarios de los GRD correspondientes para los efectos adversos moderados o graves o el coste } \\
\text { de una consulta de urgencia en atención primaria en el caso de los efectos adversos leves }\end{array}$ & 7,22 \\
\hline $\begin{array}{l}\text { 17. Coste mensual del tratamiento de soporte } \\
\text { - SLP-1L, grupo de rituximab: } 199,44 € \\
\text { - SLP-1L, grupo de observación: } 139,14 € \\
\text { - SLP-2L: } 227,25 € \\
\text { - SLP-3L: } 2.701,31 €\end{array}$ & $\begin{array}{l}\text { Panel } \\
\text { de } \\
\text { expertos }\end{array}$ \\
\hline 18. Tasas anuales de descuento para costes y utilidades: $3,5 \%[1,5-6,0 \%]^{*}$ & 18 \\
\hline
\end{tabular}

1L: primera línea de tratamiento; 2L: segunda línea de tratamiento; 3L: tercera línea de tratamiento; CHOP: ciclofosfamida, doxorubicina, vincristina, prednisona; CVP: ciclofosfamida, vincristina, prednisolona; FCM: fludarabina, ciclofosfamida, mitoxantrona; GRD: grupos relacionados por el diagnóstico; HR: hazard ratio; PVL: precio de venta del laboratorio; SLP: supervivencia libre de progresión. * Entre corchetes se indican los valores aplicados en los análisis de sensibilidad.

Estimación de las utilidades. Las utilidades se midieron como AVAC, siendo un AVAC un año de vida multiplicado por un factor de ponderación que indica la calidad de vida de la persona durante ese año. El "peso" o factor de ponderación de la calidad de un año de vida, puede ir desde el valor 0 (la muerte o un estado equivalente) hasta el valor 1 (que indica la salud perfecta). Las utilidades empleadas en el modelo (tabla 1) se tomaron de un estudio realizado en el Reino Unido con 152 pacientes con LF, a los que se administró la escala analógica visual del instrumento EQ-5D ${ }^{11,12}$. Los valores de las utilidades de los estados de salud SLP-1L, SLP-2L y progresión, estimadas mediante la tarifa de York, se indican en la tabla 1.

Duración de los ciclos, horizonte temporal y descuentos. Las transiciones entre estados se realizaron en unos periodos discretos de tiempo denominados "ciclos" que, como se dijo anteriormente, en el modelo tuvieron una duración de 1 mes $^{9}$. En el caso base del análisis, la duración máxima del tratamiento de mantenimiento con rituximab fue de 2 años (conforme al estudio PRIMA) ${ }^{7}$.

El horizonte temporal del caso base del análisis fue de 30 años (ya que se estimó que solo el $5 \%$ de la cohorte hipotética de pacientes sobreviviría a ese periodo), es decir, hasta la muerte de casi toda la cohorte ${ }^{13}$. No obstante, en el análisis de sensibilidad también se consideraron lapsos de 6, 10 y 20 años.

Para desarrollar un modelo con un seguimiento de 30 años en el caso base, fue necesario extrapolar los datos de Kaplan Meier obtenidos del ensayo clínico, utilizando diferentes distribuciones estadísticas (Weibull en el caso base; exponencial, log-logística, log-Normal, Gompertz y gamma en el análisis de sensibili(ad) $)^{14}$.

Debido a la amplia censura de la supervivencia global en el estudio PRIMA (95\% y $97 \%$ en los grupos de rituximab y observación, respectivamente) las probabilidades de progresión o de muerte en la segunda o tercera línea se obtuvieron a partir del ensayo clínico EORTC $20981^{15}$ ). Para estimar la mortalidad desde el estado de progresión, se utilizó un modelo de mínimos cuadrados ordinarios ${ }^{16}$.

La mortalidad por todas las causas en España se obtuvo a partir de las tablas de mortalidad del Instituto Nacional de Estadística ${ }^{17}$.

Se hizo un descuento anual del 3,5\% para los costes y para los beneficios 
Figura 1

Estructura del modelo de Markov para la simulación del tratamiento de mantenimiento con rituximab en el linfoma folicular que responde a la terapia de inducción en primera línea

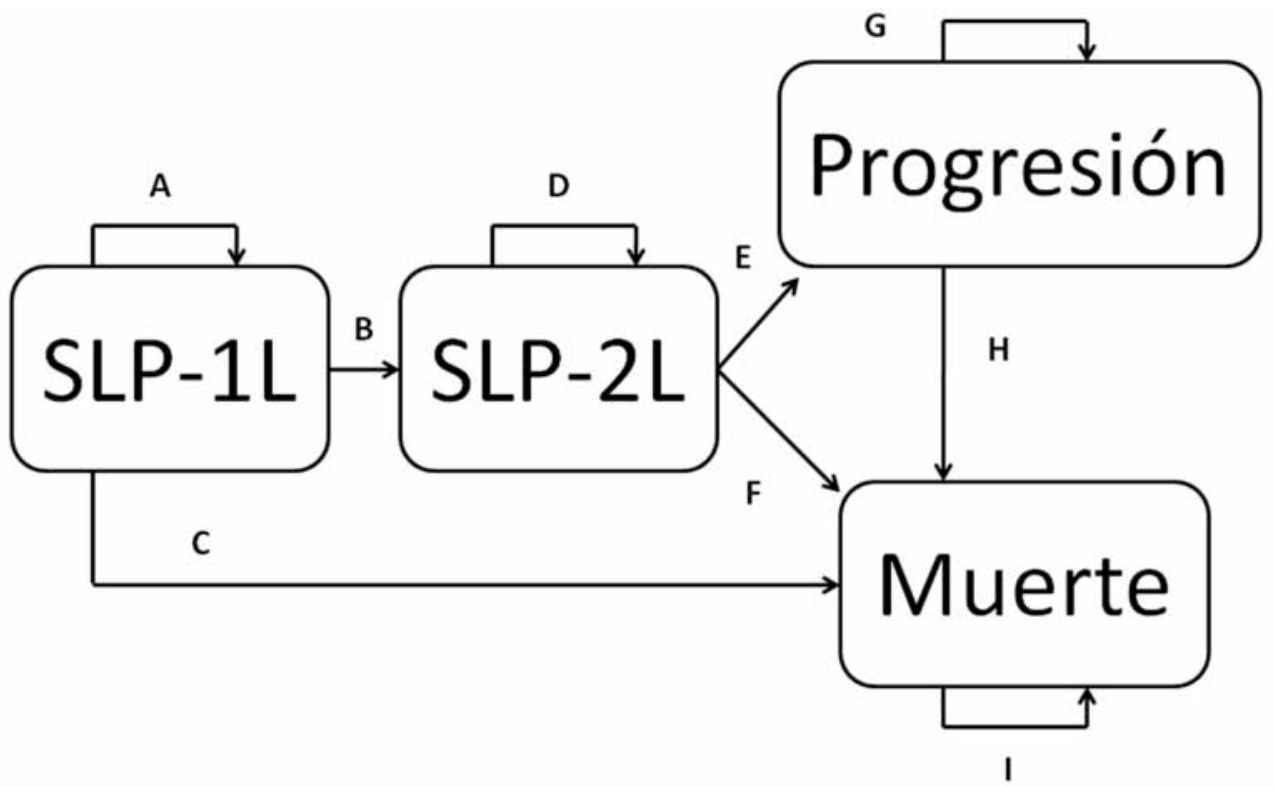

SLP-1L: supervivencia libre de progresión en pacientes que responden a la terapia de inducción en primera línea (R-CHOP, R-CVP o R-FCM) seguida de mantenimiento con rituximab u observación.

SLP-2L: supervivencia libre de progresión en pacientes que recaen después de SLP-1L y responden a una segunda línea de inducción seguida de mantenimientos con rituximab

A-C:Probabilidades de transición obtenidas a partir del estudio PRIMA.

D-I: Probabilidades de transición obtenidas a partir del estudio EORTC 20981

(AVAC, AV) según las recomendaciones del National Institute for Clinical Excellence (NICE) ${ }^{18}$ que se contabilizó en la mitad de cada ciclo.

Perspectiva del estudio y directrices seguidas. El estudio se hizo desde la perspectiva del Sistema Nacional de Salud (SNS) español, por tanto considerando únicamente los costes directos sanitarios.

Se siguieron las directrices generales para la realización de análisis de costeefectividad en España ${ }^{19}$, excepto en lo relativo a la tasa de descuento anual ya que se adoptó la recomendada por el NICE, así como las directrices publicadas por la Agencia Canadiense de Medicamentos y Tecnologías Sanitarias ${ }^{20}$, los Principios de buenas prácticas de modelización de la International Society for Pharmacoeconomics and Outcomes Research (ISPOR) ${ }^{21}$ y las recomendaciones del NICE ${ }^{18}$.

Estimación de los costes. La estimación de los costes de una enfermedad tratada con un determinado fármaco se hace mediante la identificación y cuantificación de los recursos sanitarios que conlleva y asignando a los recursos unos determinados costes unitarios. De este modo, se estimaron los costes medios para un paciente tipo con LF. Los costes de los 
recursos sanitarios utilizados en el modelo se presentan en euros $(€)$ de 2.011 .

Solo se consideraron los costes anuales a partir de la aleatorización de los pacientes al grupo de mantenimiento con rituximab o al grupo de observación, en el ensayo clínico PRIMA. Los costes unitarios se recogen en la tabla 1 .

Se analizaron cuatro tipos de costes: (i) adquisición y administración de rituximab (estimado mediante las dosis previstas $o$, en el análisis de sensibilidad, a partir de las dosis recibidas por paciente en PRIMA, para un coste unitario del hospital de día promedio de los precios públicos de 9 Comunidades Autónomas) ${ }^{7,22-30}$; (ii) acontecimientos adversos (AA), según su frecuencia de aparición con rituximab u observación en PRIMA ${ }^{7}$, mediante los costes unitarios de sus grupos relacionados por el diagnóstico (GRD) para los AA graves o moderados o el coste de una consulta de urgencia en atención primaria en el caso de los AA leves, obtenidos a partir de los precios públicos de la Comunidad de Madrid ${ }^{22}$; (iii) el coste del tratamiento de soporte en los estados de SLP en primera, segunda o tercera línea, o en progresión, a partir de la estimación del consumo de recursos sanitarios efectuada por un panel de Oncohematólogos españoles; y (iv) el coste de los esquemas de quimioterapia empleados en el tratamiento de inducción del $\mathrm{LF}^{31-35}$ ).

Para calcular el coste de adquisición de rituximab y de los esquemas de inducción, se utilizaron los precios de venta del laboratorio (PVL) descontados según lo dispuesto en el Real Decreto-Ley $8 / 2010^{36}$ y las pautas posológicas del estudio $\mathrm{PRIMA}^{7}$ para un peso corporal medio de $64,3 \mathrm{~kg}$ y una estatura media de $161,5 \mathrm{~cm}$ (que se corresponden con una superficie corporal aproximada de $1,7 \mathrm{~m} 2$ ), de acuer- do con los datos estadísticos del Ministerio de Sanidad y Consumo sobre la talla y el peso de los españoles ${ }^{37}$. La edad de la cohorte de pacientes con LF (60 años) se estableció conforme a la mediana de edad en el diagnóstico del LF de 286 pacientes españoles incluidos en el Registro Español de Linfomas $^{38}$ (tabla 1).

Análisis de sensibilidad. Para comprobar la estabilidad de los resultados y la consistencia de las estimaciones efectuadas, se hicieron análisis de sensibilidad determinísticos para las siguientes variables: (i) el tipo de distribución estadística (exponencial, loglogística, log-normal, Gompertz o Gamma); (ii) el horizonte temporal de la simulación (10 o 20 años y análisis umbral); (iii) con reutilización del fármaco sobrante; (iv) con las dosis de rituximab empleadas en el estudio PRIMA; (v) $\pm 20 \%$ del coste del tratamiento de soporte en SLP y progresión; (vi) $\pm 20 \%$ del coste de la administración de rituximab; (vii) $\pm 20 \%$ del coste de los AA; (viii) $\pm 10 \%$ de las utilidades de los estados de salud; (ix) para tasas de descuento anuales de costes y beneficios del $1,5 \%$ y $6 \%$; y, finalmente, (x) sin aplicar descuentos a los PVL de los fármacos.

Se hizo un análisis de sensibilidad probabilístico mediante una simulación de Monte Carlo, para comprobar la estabilidad del modelo ${ }^{39}$.

\section{RESULTADOS}

\section{Análisis de costes}

En el caso base del análisis determinístico, el coste promedio por paciente en mantenimiento con rituximab fue de $96.814 €$ y de $88.416 €$ en el grupo de observación, con un coste incremental con rituximab de $8.398 €$ (tabla 2 ). 
Tabla 2

Resultados por paciente del caso base*: análisis determinístico y probabilístico (€ de 2.011)

\begin{tabular}{|c|c|c|c|c|c|c|}
\hline \multirow[b]{2}{*}{ Item } & \multicolumn{3}{|c|}{ Deterministico } & \multicolumn{3}{|c|}{ Probabilistico } \\
\hline & Rituximab & Observación & Diferencia & Rituximab & Observación & Diferencia \\
\hline \multicolumn{7}{|l|}{ Años de vida } \\
\hline En SLP & 8,567 & 6,671 & 1,896 & 8,571 & 6,679 & 1,892 \\
\hline En Progresión & 1,208 & 1,621 & $-0,413$ & 1,209 & 1,623 & $-0,414$ \\
\hline En total & 9,775 & 8,292 & 1,483 & 9,78 & 8,302 & 1,478 \\
\hline \multicolumn{7}{|c|}{$\begin{array}{l}\text { Años de vida ajustados por su calidad } \\
\text { (AVAC) }\end{array}$} \\
\hline En SLP & 7,275 & 5,676 & 1,599 & 7,277 & 5,68 & 1,597 \\
\hline En Progresión & 0,749 & 1,005 & $-0,256$ & 0,75 & 1,007 & $-0,257$ \\
\hline En total & 8,024 & 6,681 & 1,343 & 8,027 & 6,687 & 1,34 \\
\hline Costes totales & $96.814 €$ & $88.416 €$ & $8.398 €$ & $97.246 €$ & $87.141 €$ & $10.105 €$ \\
\hline Coste por año de vida ganado & \multicolumn{3}{|c|}{$5.663 €$} & \multicolumn{3}{|c|}{$6.837 €$} \\
\hline Coste de ganar un AVAC & \multicolumn{3}{|c|}{$6.253 €$} & \multicolumn{3}{|c|}{$7.541 €$} \\
\hline Beneficio neto monetario & \multicolumn{3}{|c|}{36.054} & \multicolumn{3}{|c|}{39.603} \\
\hline
\end{tabular}

AVAC: años de vida ajustados por su calidad; SLP: supervivencia libre de progresión.

* Función de Weibull. Horizonte temporal: 30 años. Sin reutilización de los viales

\section{Análisis de efectividad}

En el caso base del análisis determinístico, se obtuvieron más años de vida $(1,483)$ y más AVAC $(1,343)$ por paciente en mantenimiento con rituximab que con observación (tabla 2).

\section{Coste-efectividad incremental}

En el caso base del análisis determinístico, para un horizonte temporal de 30 años, el coste por AVG con el tratamiento más efectivo (mantenimiento con rituximab) fue de $5.663 €$, y el coste por AVAC ganado fue de $6.253 €$ (tabla 2 ).

\section{Análisis de sensibilidad}

Los resultados de los análisis de sensibilidad oscilaron entre $4.222 €$ por AVG (tasa de descuento anual del 1,5\%) y $8.766 €$ por AVAC ganado (horizonte temporal de 10 años). El mantenimiento con rituximab es coste-efectivo desde horizontes temporales muy cortos (por debajo incluso del tiempo habitual en el que transcurre la patología) (tabla 3).

El análisis de sensibilidad probabilístico confirmó la estabilidad del caso base, con un coste por AVAC ganado de 7.541 $€$. Rituximab fue coste-efectivo en el $100 \%$ de las simulaciones efectuadas (figura 2). Como puede apreciarse en la curva de aceptabilidad del coste-efectividad incremental, el mantenimiento con rituximab sería coste-efectivo a partir de una disponibilidad a pagar de $10.000 €$ por AVAC ganado (figura 3). 
Tabla 3

Análisis de sensibilidad determinísticos del modelo de Markov (€ de 2.011)

\begin{tabular}{|c|c|c|c|}
\hline Escenarios & Variaciones & $\begin{array}{l}\text { Coste por } \\
\text { AVG } \\
(\epsilon)\end{array}$ & $\begin{array}{c}\text { Coste por } \\
\text { AVAC ganado } \\
(€)\end{array}$ \\
\hline Caso base & - & 5.663 & 6.253 \\
\hline \multicolumn{4}{|l|}{ Análisis de sensibilidad (supuestos del caso base) } \\
\hline Con reutilización del fármaco sobrante (sin reutilización) & - & 6.488 & 7.159 \\
\hline Dosis de rituximab del estudio PRIMA (dosis prevista) & - & 6.331 & 6.986 \\
\hline \multirow{2}{*}{$\begin{array}{l}\text { Coste del tratamiento de soporte en SLP y Progresión } \\
\text { promedio }\end{array}$} & $-20 \%$ & 6.259 & 6.906 \\
\hline & $20 \%$ & 5.077 & 5.602 \\
\hline \multirow{2}{*}{ Costes mensuales de administración de Rituximab $(263,74 €)$} & $-20 \%$ & 5.396 & 5.954 \\
\hline & $20 \%$ & 5.939 & 6.553 \\
\hline \multirow{2}{*}{ Costes de los acontecimientos adversos (promedio) } & $-20 \%$ & 5.578 & 6.154 \\
\hline & $20 \%$ & 5.758 & 6.353 \\
\hline \multirow{2}{*}{$\begin{array}{l}\text { Utilidades de los estados de salud } \\
\text { (valores publicados) }\end{array}$} & $-10 \%$ & - & 6.949 \\
\hline & $10 \%$ & - & 5.685 \\
\hline \multirow{2}{*}{ Tasa de descuento anual para costes y utilidades $(3,5 \%)$} & $1,50 \%$ & 4.222 & 4.716 \\
\hline & $6,00 \%$ & 7.824 & 8.499 \\
\hline \multirow{3}{*}{ Horizonte temporal del modelo ( 30 años) } & 10 años & 8.706 & 8.766 \\
\hline & 20 años & 5.885 & 6.451 \\
\hline & Umbral: 5,7 años & 24.624 & 29.998 \\
\hline \multirow{5}{*}{$\begin{array}{l}\text { Otras funciones para estimar las probabilidades de transición } \\
\text { (Weibull) }\end{array}$} & Exponencial & 5.684 & 6.213 \\
\hline & Log-Logistica & 5.699 & 6.197 \\
\hline & Log-Normal & 6.810 & 7.403 \\
\hline & Gompertz & 5.640 & 6.216 \\
\hline & Gamma & 6.395 & 6.962 \\
\hline Sin descuento del PVL de los fármacos (con descuento) & - & 6.496 & 7.157 \\
\hline
\end{tabular}

AVAC: años de vida ajustados por su calidad; AVG: año de vida ganado; SLP: supervivencia libre de progresión. 
Figura 2

Resultado del análisis probabilístico (1.000 análisis; disponibilidad a pagar de 30.000 euros por AVAC ganado)

$$
y=30000 x
$$

0
0
0
0
0
0
0
$\frac{0}{0}$
$\frac{0}{0}$
$\frac{1}{0}$
$\frac{0}{0}$

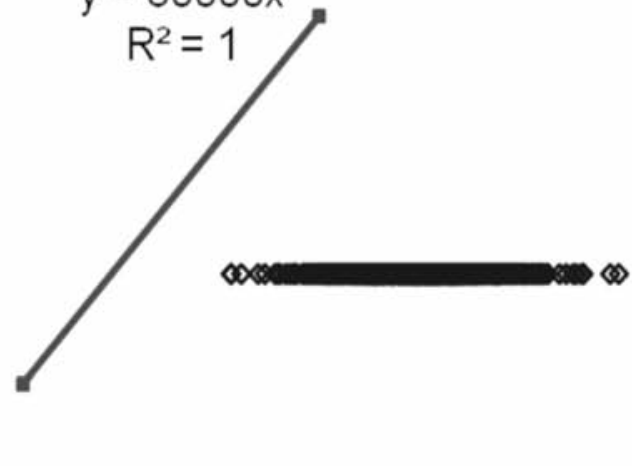

Diferencia de AVAC

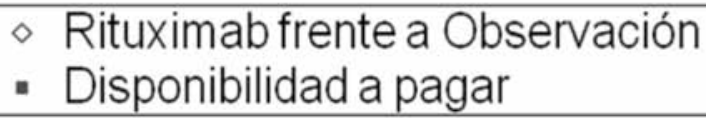

Figura 3

Curva de aceptabilidad de coste-efectividad (el esquema con rituximab fue coste-efectivo a partir de una disponibilidad a pagar de $\mathbf{1 0 . 0 0 0} €$ ). Probabilidad de coste-efectividad del $\mathbf{1 0 0 \%}$ para una disponibilidad a pagar de $\mathbf{3 0 . 0 0 0} €$

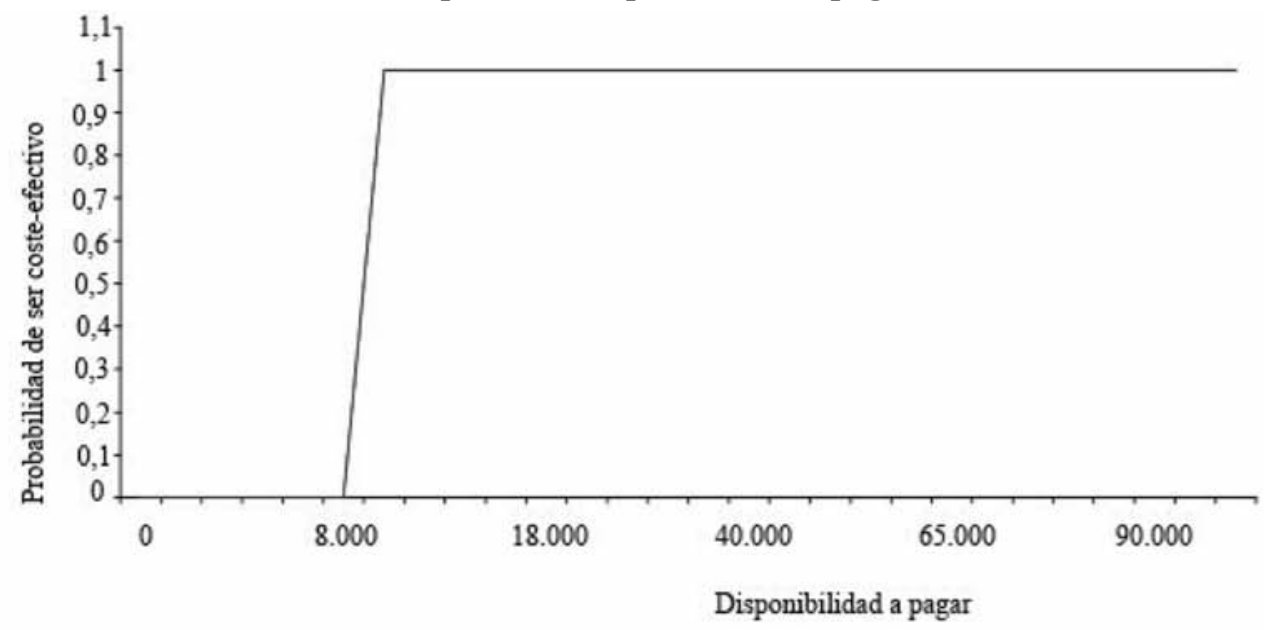

- Rituximab frente a observación 


\section{DISCUSIÓN}

De acuerdo con los resultados del modelo, el tratamiento de mantenimiento con rituximab del linfoma folicular que responde a la inducción en primera línea es coste-efectivo en comparación con la observación de los pacientes.

En la valoración de estos resultados debemos considerar, en primer lugar, que se trata de un modelo teórico (que es, por definición, una simulación simplificada de la realidad) basado no obstante en los resultados de ensayos clínicos aleatorizados que compararon directamente las opciones estudiadas, con un diseño no pragmático. Por este motivo, cobra especial importancia la validación del modelo por un panel de 3 expertos oncohematólogos españoles que estimaron la utilización de recursos en la práctica clínica en nuestro país, por lo que sus resultados deben considerarse como estimaciones válidas para pacientes con las características de los incluidos en el análisis, pudiendo ser útiles como una herramienta para la toma de decisiones en la práctica clínica ${ }^{6}$.

Un aspecto que debe mencionarse es el hecho de que las utilidades de los estados de salud empleadas en el modelo se obtuvieron de un estudio del Reino Unido. Aunque las preferencias por los estados de salud pueden variar entre países debido a factores culturales ${ }^{20}$, este riesgo es menor cuando se comparan países de similar nivel socioeconómico. A este respecto, debe comentarse además que en un estudio basado en 83.000 evaluaciones de 44 estados de salud de EuroQol (EQ-5D), realizado en seis países europeos incluyendo España, se constató una mayor variabilidad entre individuos que entre países $^{40}$.

Por otra parte, también debe tenerse en cuenta que, gracias al modelo de Markov, fue posible estimar de una manera más "realista" que con un modelo determinístico puro la evolución de la enfermedad a lo largo de 30 años ${ }^{10}$. Del mismo modo, deben señalarse como "fortalezas" del modelo que la estimación de los costes unitarios de los recursos sanitarios y de los acontecimientos adversos se hizo a partir de fuentes españolas ${ }^{22-30}$ empleando los GRD y que las utilidades se obtuvieron mediante una metodología adecuada, a partir de pacientes con linfoma folicular.

Para intentar minimizar las limitaciones del modelo, en el caso base se tomaron premisas conservadoras y se hicieron análisis de sensibilidad determinísticos y probabilísticos, que confirmaron la estabilidad de los valores considerados.

Únicamente se ha identificado un estudio de farmacoeconomía en el tratamiento de mantenimiento con rituximab del LF que responde a la inducción en primera línea ${ }^{9}$, realizado en el Reino Unido con el mismo modelo utilizado en el presente estudio y con resultados del mismo signo, obteniéndose un coste por AVG y por AVAC ganado de 14.712 y 15.983 libras esterlinas, respectivamente (unos 16.600 y $18.100 €)$. El coste-efectividad incremental no excedió de las 21.155 libras por AVAC ganado en el análisis probabilístico (unos $24.000 €$ ). Las diferencias cuantitativas entre los resultados deben achacarse tanto a las variaciones entre los sistemas sanitarios como a los distintos costes unitarios de ambos países.

Aunque la metodología seguida en el presente análisis es adecuada, no obstante sería de interés la realización de ensayos clínicos pragmáticos y aleatorizados, en los que se comparasen directamente la eficacia, las utilidades, la tolerancia y el consumo de recursos sanitarios de las alternativas terapéuticas evaluadas, para confirmar los resultados de este análisis. Mientras tanto, de acuerdo con los resultados del modelo, puede concluirse que, 
en comparación con la opción de "observación", el tratamiento de mantenimiento del linfoma folicular que responde a la inducción en primera línea con rituximab (además de mejorar la supervivencia libre de progresión) proporcionaría más años de vida ajustados por su calidad, a un coste medio por AVAC de unos 5.000-7.000 €, por lo que puede considerarse una opción coste-efectiva.

\section{BIBLIOGRAFÍA}

1. Rodríguez-Abreu D, Llanos M, Provencio M, Rueda A, Isla D. SEOM clinical guidelines for the treatment of follicular non-Hodgkin's lymphoma. Clin Transl Oncol. 2010; 12: 760-4.

2. Fisher RI, LeBlanc M, Press OW, et al. New treatment options have changed the survival of patients with follicular lymphoma. J Clin Oncol. 2005;23:8447-52

3. Ardeshna KM, Smith P, Norton A, et al. Long-term effect of a watch and wait policy versus immediate systemic treatment for asymptomatic advanced-stage non-Hodgkin lymphoma: a randomised controlled trial. Lancet .2003; 362: 516-22.

4. Czuczman MS. Controversies in follicular lymphoma: "who, what, when, where, and why?" (not necessarily in that order!). Hematology Am Soc Hematol Educ Program. 2006:303-10.

5. Keating GM. Rituximab: a review of its use in chronic lymphocytic leukaemia, low-grade or follicular lymphoma and diffuse large B-cell lymphoma. Drugs. 2010; 70: 1445-76.

6. Ficha técnica o Resumen de características del producto. MabThera $100 \mathrm{mg}$ concentrado para solución para perfusión. [citado: 07/2011]. Disponible en: https://sinaem4.agemed.es/consaem/fichasTecnicas.d $\mathrm{o}$ ?metodo $=$ detalleForm $\&$ version $=$ new

7. Salles G, Seymour JF, Offner F, López-Guillermo A, Belada D, Xerri L, et al. Rituximab maintenance for 2 years in patients with high tumour burden follicular lymphoma responding to rituximab plus chemotherapy (PRIMA): a phase 3, randomised controlled trial. Lancet $2011 ; 377: 42-51$.

8. Rubio-Terrés C, Sacristán JA, Badía X, Cobo E, García Alonso F, por el Grupo ECOMED. Métodos utilizados para realizar evaluaciones económicas de intervenciones sanitarias. Med Clín (Barc). 2004; 122: 578-83.
9. Papadakis K, Follows GA, Boyer J, Bashir Z, Ball P, Aultman R, et al. Cost Effectiveness Analysis of Rituximab Maintenance In Patients with Untreated High Tumour Burden Follicular Lymphoma After Response to Immunochemotherapy: A UK National Healthcare Services Perspective. 52nd ASH Annual Meeting and Exposition (December 4-7, 2010). [citado: 06/2011]. Disponible en: http://ash.confex.com/ash/2010/webprogram/Paper 32784.html

10. Rubio-Terrés C, Echevarría A. La herramienta clave: modelos de Markov. Pharmacoeconomics-Spanish Research Articles. 2006; 3 (Suppl 2): 71-78.

11. Wild D, Tabberer M. Utility values in follicular lymphoma. Oxford: Oxford Outcomes; 2005.

12. Pettengell R, Donatti C, Hoskin P, Poynton C, Kettle PJ, Hancock B, Johnson S, Dyer MJ, Rule S, Walker M, Wild D. The impact of follicular lymphoma on healthrelated quality of life. Ann Oncol. 2008; 19: 570-6.

13. Horning SJ. Natural history of and therapy for the indolent non-Hodgkin's lymphomas. Semin Oncol. 1993; 20: 75-88.

14. Chapman and Hall. Applied Survival Analysis. New York: John Wiley \& Sons, Inc; 1984.

15. van Oers MH, Van Glabbeke M, Giurgea L et al. Rituximab maintenance treatment of relapsed/resistant follicular non-Hodgkin lymphoma: Long-term outcome of the EORT 2098 phase III randomized intergroup study. JCO. 2010; 28: 2853-8.

16. Sánchez JM, Medina A. Modelos de supervivencia adecuados para análisis actuariales de mortalidad. Universidad de Oviedo. XI Jornadas ASEPUMA. Oviedo, 11 y 12 de septiembre de 2003 .

17. Instituto Nacional de Estadística. Tablas de mortalidad de la población de España por año, sexo, edad y funciones. INEbase. [citado: 11/2010]. Disponible en: http://www.ine.es/jaxi/tabla.do

18. NICE. Guide to the methods of technology appraisal. London: National Institute for Clinical Excellence, April 2004.

19. López Bastida J, Oliva J, Antoñanzas F, García Altés A, Gisbert R, Mar J, Puig Junoy J. Propuesta de guía para la evaluación económica aplicada a las tecnologías sanitarias. Gac Sanit. 2010;24(2):154-70.

20. Guideline for the economic evaluation of health technologies: Canada (3rd edition). Ottawa: Canadian Agency for Drugs and Technologies in Health; 2006. 
21. Weinstein MC, O’Brien B, Hornberger J, Jackson J, Johannesson M, McCabe C, et al. Principles of good practice for decision analytic modeling in health-care evaluation: report of the ISPOR Task Force on good research practices-modeling studies. Value Health. 2003; 6: 9-17.

22. Orden 629/2009, de 31 de agosto, por la que se fijan los precios públicos por la prestación de los servicios y actividades de naturaleza sanitaria de la red de centros de la Comunidad de Madrid. BOCM No 215 de 10/09/2009.

23. Decreto $87 / 2009$, de 29 de julio, por el que se establecen los precios públicos a aplicar por el Servicio de Salud del Principado de Asturias por la prestación de servicios sanitarios.BOPA No 186 de 11/08/2009.

24. Orden del consejero de Salud y Consumo, de 11 de septiembre de 2008, de modificación de la Orden del consejera de Salud y Consumo de 22 de diciembre de 2006, por la que se establecen los precios públicos que han de aplicar los centros sanitarios de la Red pública de las Islas Baleares por la prestación de servicios sanitarios cuando haya terceros obligados al pago o usuarios sin derecho a la asistencia sanitaria de la Seguridad Social. BOIB num 131 de 18/09/2008.

25. Orden SAN/6/2010, de 4 de junio, por la que se modifica la Orden SAN/8/2008, de 4 de abril, por la que se fi jan las cuantías de los precios públicos de los servicios sanitarios prestados por el Servicio Cántabro de Salud. BOC num 117 de 18/06/2010.

26. Resolución SLT/383/2009, de 21 de enero, sobre la revisión de precios públicos correspondientes a los servicios sanitarios que presta el Instituto Catalán de la Salud. DOGC num 5325 de 24/02/2009.

27. Decreto $21 / 2009$, de 13 de febrero, por el que se modifican las cuantías de los precios públicos correspondientes a los servicios sanitarios del Servicio Extremeño de Salud establecidos en el Decreto 272/2005, de 27 de diciembre, por el que se establecen y regulan los precios públicos correspondientes a los servicios sanitarios del Servicio Extremeño de Salud. DOE num 34 de 19702/2009.

28. Resolución 882/2010, de 3 de mayo, del Director Gerente del Servicio Navarro de Salud, por la que se actualizan las tarifas por prestación de servicios en los centros y establecimientos asistenciales del Servicio Navarro de Salud. BCFN num 71 de 11/06/2010.

29. Resolución $n^{\circ} 143$, de 4 de mayo de 2010, del Consejero de Salud por la que se dispone la publicación de las tarifas por servicios sanitarios prestados a particulares en los centros del Servicio Riojano de Salud. BOLR num 57 , de $12 / 05 / 2010$.
30. Decreto Legislativo $1 / 2005$, de 25 de febrero, por el que se aprueba el Texto Refundido de la Ley de Tasas de la Generalitat. DOGV num 4971 de 22/03/2005.

31. van Oers MH, Klasa R, Marcus RE, et al: Rituximab maintenance improves clinical outcome of relapsed/resistant follicular non-Hodgkin lymphoma in patients both with and without rituximab during induction: Results of a prospective randomized phase 3 intergroup trial. Blood. 2006; 108: 3295-3301.

32. Grupo Andaluz de Neoplasias Linfoides. Guía para el tratamiento del linfoma folicular. Mayo de 2009. [citado: 19 de noviembre de 2010]. [citado: 19/11/2010] Disponible en: http://www.aahh.org/data/archivos/ LINFOMA\%20FOLICULAR\%20guia\%20andalucia\%20mayo09.pdf

33. Agencia Española de Medicamentos y Productos Sanitarios. Centro de Información online de Medicamentos de la AEMPS. Disponible en: https://sinaem4.agemed.es/ consaem/fichasTecnicas.do? metodo $=$ detalleForm $\&$ version $=$ new

34. Hornberger J, Reyes C, Lubeck D, Valente N. Economic evaluation of rituximab plus cyclophosphamide, vincristine and prednisolone for advanced follicular lymphoma. Leuk. Lymphoma. 2008; 49: 227-36.

35. Forstpointer R, Dreyling M, Repp R, Hermann S, Hänel A, Metzner E, et al. The addition of rituximab to a combination of fludarabine, cyclophosphamide, mitoxantrone (FCM) significantly increases the response rate and prolongs survival as compared with FCM alone in patients with relapsed and refractory follicular and mantle cell lymphomas: results of a prospective randomized study of the German LowGrade Lymphoma Study Group. Blood. 2004; 104: 3064-71.

36. Listado elaborado por el Consejo General con los medicamentos afectados por el Real Decreto-ley 8/2010, dispensables en Oficina de Farmacia (excluidos envases clínicos y hospitalarios) en estado de alta. [citado: junio de 2011]Disponible en: www.portalfarma.com .

37. Ministerio de Sanidad y Consumo. Talla y peso medios de los españoles (2001). [citado: diciembre de 2010].Disponible en: http://www.msc.es/gl/estadEstudios/estadisticas/docs/CCap._1_Poblaci_n.xls\#'Talla y peso'!A1.

38. Gabriel R, Arranz R, Damiano S, Acevedo A, Aguado B, Fernández JM. Registro Español de Linfomas. Madrid: Fundación Leucemia y Linfoma, 2005 . 
39. Rubio-Terrés C, Cobo E, Sacristán JA, Prieto L, del Llano J, Badia X, por el Grupo ECOMED. Análisis de la incertidumbre en las evaluaciones económicas de intervenciones sanitarias. Med Clin (Barc). 2004; 122: 668-674.

40 . Greiner W, Weijnen T, Nieuwenhuizen M, Oppe S, Badia X, Busschbach J, et al. A single European currency for EQ-5D health states. Results from a six-country study. Eur J Health Econ. 2003; 4: 22231 